\section{References}

1. Kato A, Kudo S, Matsumoto K, et al. Bronchial artery embolization for hemoptysis due to benign diseases: immediate and long-term results. Cardiovasc Intervent Radiol. 2000;23:351-7.

2. Mal H, Rullon I, Mellot F, et al. Immediate and long-term results of bronchial artery embolization for life-threatening hemoptysis. Chest. 1999;115:996-1001
3. Moore LB, Mc Wey RE, Vujic I. Massive hemoptysis: control by embolization of the thyrocervical trunk. Radiology. 1986;161:173-4.

4. Sancho C, Escalante E, Dominguez J, et al. Embolization of bronchial arteries of anomalous origin. Cardiovasc Intervent Radiol. 1998;21: 300-4.

5. Cohen AM, Antoun BW, Stern RC. Left thyrocervical trunk bronchial artery supplying right lung: source of recurrent hemoptysis in cystic fibrosis. AJR Am J Roentgenol. 1992;158:1131-3.

\title{
Liposarcoma of the pleural cavity: A case report
}

\author{
Chuanliang Peng, MD, Xiaogang Zhao, MD, Xiaopeng Dong, MD, and
}

Xingtao Jiang, MD, Jinan, China

W

e present a case of a large liposarcoma in the left pleural cavity. Pleural liposarcomas are rare; only a few cases have been reported in the English literature. We describe a 56-year-old woman who had dyspnea. A computed tomographic (CT) scan of the chest showed a large fat density mass in the left pleural cavity. Thoracotomy was performed, and postoperative chemotherapy was given 1 month later. She has had a good quality of life for 1.5 years without recurrence.

Liposarcoma is the most common mesenchymal sarcoma in adult life and usually occurs in the retroperitoneum and deep soft tissues of the trunk and extremities, representing approximately $20 \%$ of mesenchymal neoplasms. The first case of primary pleural liposarcoma was reported by Ackerman and Wheeler ${ }^{1}$ in 1942. There are only 14 cases reported in the English literature up to the present. ${ }^{2-4}$ We report an additional case.

\section{Clinical Summary}

A 56-year-old woman with a 3-year history of progressive dyspnea, was urgently admitted to our hospital in January 2005. CT showed that the tumor, which was detected as a large and lowdensity area in the left pleural cavity, occupied the entire left pleural cavity, the mediastinum, and part of the right pleural cavity (Figure 1). At admission, the patient presented with a pulse rate of 120 beats/min, a blood pressure of $93 / 55 \mathrm{~mm} \mathrm{Hg}$, a breath rate of 30 breaths/min, and an oxygen saturation of approximately

\footnotetext{
From the Department of Thoracic Surgery, Second Hospital of Shandong University, Jinan, China.

Received for publication Dec 7, 2006; accepted for publication Dec 12, 2006.

Address for reprints: Xiaogang Zhao, The Second Hospital of Shandong University, Thoracic Department, 247 Beiyuan Street, Jinan, Shandong, 250033 China (E-mail: menglin2002@163.com).

J Thorac Cardiovasc Surg 2007;133:1108-9

0022-5223/\$32.00

Copyright $\odot 2007$ by The American Association for Thoracic Surgery doi:10.1016/j.jtcvs.2006.12.014
}

70\%. We preoperatively diagnosed liposarcoma with unknown origin.

The patient underwent a tumor resection with thoracotomy. Her vital signs worsened at the beginning of the operation. We quickly removed part of the tumor from the pleural

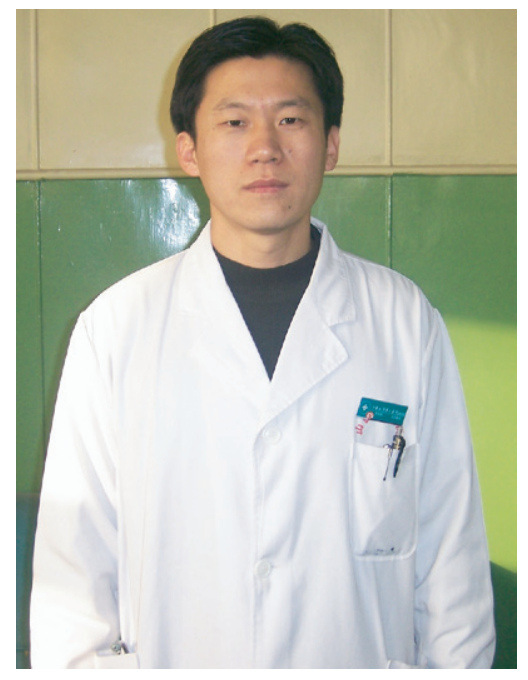

Dr Peng cavity to reduce the pressure on the great vessels and heart. When the patient's vital signs improved, we operated step by step, and the soft tumor was completely removed. The total weight of the resected tumor mass was $3000 \mathrm{~g}$. The histologic examination revealed a well-differentiated liposarcoma (Figure 2).

The postoperative course was uneventful. The patient recovered with no major complication and was discharged from the hospital 11 days after the operation. Postoperative chemotherapy

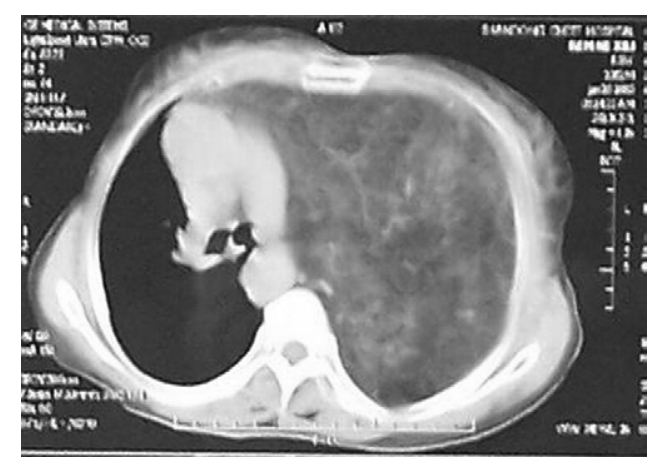

Figure 1. CT scan of the chest showing a large fat density mass in the left pleural cavity and mediastinum, and vascular displacement. 


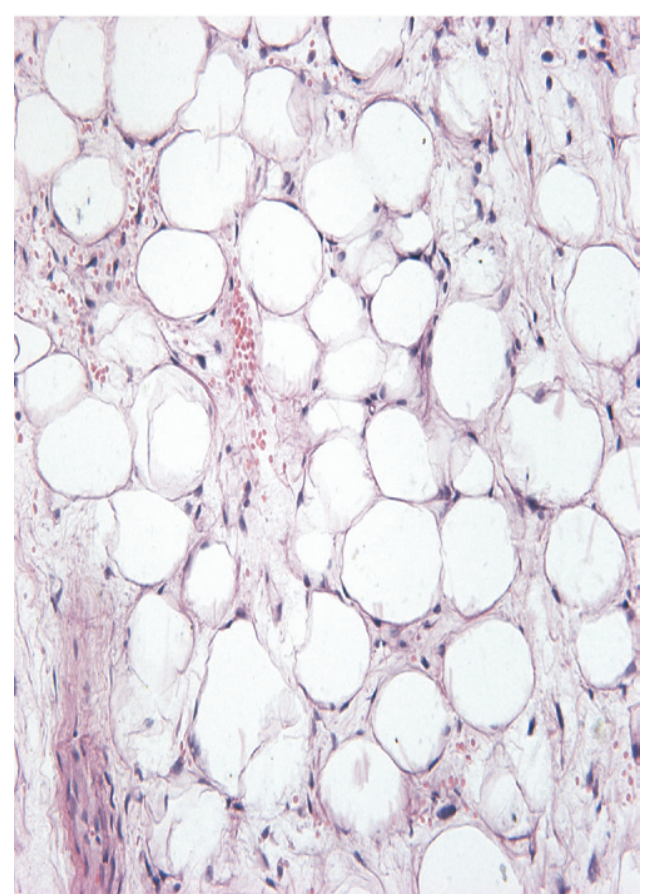

Figure 2. Capillary network, different sizes of lipocytes, fibrous interval, and scattered lipoblast in well-differentiated liposarcoma (hematoxylin-eosin, magnification $75 \times$ ).

( 1 course) was given 1 month later. She has had a good quality of life for 1.5 years without recurrence.

\section{Discussion}

Including our case presented here, 15 cases of primary liposarcoma of the pleural cavity have been reported in the English literature. These cases included 10 men and 5 women, aged 19 to 80 years, with an average age of 50 years. The tumors were located in the right pleural cavity in 5 patients and in the left pleural cavity in 9 patients (1 was unknown). The weight of the tumors ranged from
0.54 to $3000 \mathrm{~g}$. In these cases, the clinical symptoms of pleural liposarcoma were pneumonia, bronchitis, pleural effusion, shortness of breath, cough, dyspnea on exertion, and chest pain. Surgical resection seemed to be the most common form of treatment; 11 patients underwent thoracotomy, 4 patients were given adjuvant radiation therapy, and 2 patients were given adjuvant chemotherapy. Our case and the previous cases reported in the English literature demonstrate that the most common histologic subtypes of liposarcoma in the pleura appear to be well differentiated and myxoid (4 well differentiated and 6 myxoid).

Liposarcoma is thought to be derived from residual rests of primitive mesenchymal tissue; pleural liposarcoma likely arises in a similar manner. ${ }^{3}$ It is the second most common soft tissue sarcoma among adults. The tumor grows slowly within the pleural cavity, usually causing no symptoms or slight dyspnea. Heavy dyspnea will appear when the tumor reaches a certain size, oppressing the lung and causing pulmonary atelectasis and a shift of the mediastinum. The patients always have a long period of time before they go to a hospital or see a doctor. Although CT imaging of the chest revealed the mass, the origin was uncertain. For many doctors, it is difficult to confirm the origin of such a large tumor preoperatively; operative information and a histologic examination are necessary. In this case, we emphasize that although the preoperative diagnosis was not accurate, we successfully performed an urgent operation.

It is difficult to predict the treatment outcomes and identify the prognostic factors because of the few studies available; however, operation was a priority.

\section{References}

1. Ackerman LV, Wheeler P. Liposarcoma. South Med J. 1942;35: 156-9.

2. Wong WW, Pluth JR, Grado GL, et al. Liposarcoma of the pleura. Mayo Clin Proc. 1994;69:882-5.

3. Okby NT, Travis WD. Liposarcoma of the pleural cavity: clinical and pathologic features of 4 cases with a review of the literature. Arch Pathol Lab Med. 2000;124:699-703.

4. Minniti A, Montaundon M, Jougon J, et al. Liposarcoma of the pleural cavity. An exceptional tumor. Monaldi Arch Chest Dis. 2005; 63:170-2. 